\title{
COVID-19 severity declines over time
}

\author{
Christian Gortazar ${ }^{1}$, Francisco Rodríguez del Río ${ }^{2}$, Dolores Hervás ${ }^{3}$, Lucas Dominguez ${ }^{4}$, \\ and Jose de la Fuente ${ }^{1}$ \\ ${ }^{1}$ IREC \\ ${ }^{2}$ Servicio de Salud, Horcajo de Los Montes, Ciudad Real \\ ${ }^{3}$ Servicio de Salud, Horcajo de Los Montes \\ ${ }^{4}$ Universidad Complutense de Madrid
}

June 10, 2020

\begin{abstract}
The first SARS-CoV-2 pandemic has affected most countries causing hundreds of thousands of deaths. Evolution of the pandemic may involve endemicity with or without significant secondary waves. We observed that disease severity $(\mathrm{p}=0.006)$ and duration of symptoms $(\mathrm{p}<0.001)$ declined over time in an isolated rural community. This decline was not driven by changes in patient age structure nor by changes in temperature or precipitations. The underlying mechanism may be relevant for COVID-19 management.
\end{abstract}

\section{Introduction}

After the first pandemic wave of coronavirus disease 19 (COVID-19) caused by severe acute respiratory syndrome coronavirus 2 (SARS-CoV-2), there are fears for a second large wave like what happened with the $1918 \mathrm{flu}$ (Shanks et al., 2011). In low-affected countries, emergence of new COVID-19 foci is likely to occur after relaxation of social distancing interventions (e.g. South Korea). However, in severely affected countries or regions such as Hubei in China, northern Italy, or Spain data available to date (as of June 9, 2020) indicate a strong decline in disease incidence after the initial epidemic, followed by occasional peaks but without recording a second large wave. Observations from a severely affected rural village in Spain provide additional insights.

\section{Material and methods}

We recorded 53 COVID-19 cases in an isolated community (883 inhabitants in 2019) in the high disease prevalence Ciudad Real province, Spain, from the first case detected on March 3, 2020, to the last one on May 9, 2020. Case definition included bilateral pneumonia, often with loss of smell and taste (Wang et al., 2020; Huang et al., 2019). Fifty-three cases were classified as moderate, severe or very severe (score 1, 2 and 3 , respectively) and duration of symptoms was recorded as another proxy for severity (Ding et al., 2020). SARS-CoV-2 was confirmed by RT-PCR in 22 patients. Cases were representative of the general population (mean case age, 54 years-old, 30 males, 23 females; mean population age, 49 years-old). Patients without bilateral pneumonia were excluded.

The data that support the findings of this study are available from the corresponding author upon reasonable request.

\section{Results and discussion}

The risk of bilateral pneumonia (Fisher's exact test; 31-530 older vs 22-322 younger, $\mathrm{p}>0.05$ ) and the duration of symptoms (ANOVA p >0.05) did not increase with age although, as expected, older patients presented 
higher disease severity (mean 2.59 \pm 0.13 ) than younger ones (mean 1.58 \pm 0.11 ; U-test, $\mathrm{p}<0.001$ )(Wu et al., 2020a; Zhang et al., 2020). However, the mean of both disease severity (U-test, $\mathrm{p}=0.006$ ) and duration of symptoms (ANOVA, $\mathrm{p}<0.001$ ) declined over time. This decline was unexpected and was not driven by changes in patient age structure (Figure 1). It was also not driven by changes in temperature or precipitations (Wu et al., 2020b; see supplementary information file). From the initial outbreak period (weeks 10 to 12 , $2020 ; n=36$ cases) to the advanced period (weeks 13 to $19 ; n=17$ cases) the mean severity declined by $34 \%$ and $21 \%$ in younger and older patients, respectively. The mean duration of symptoms (21 days, range 4-67) was $59 \%$ and $60 \%$ shorter in the advanced period in younger and older patients, respectively, as compared to the initial period.

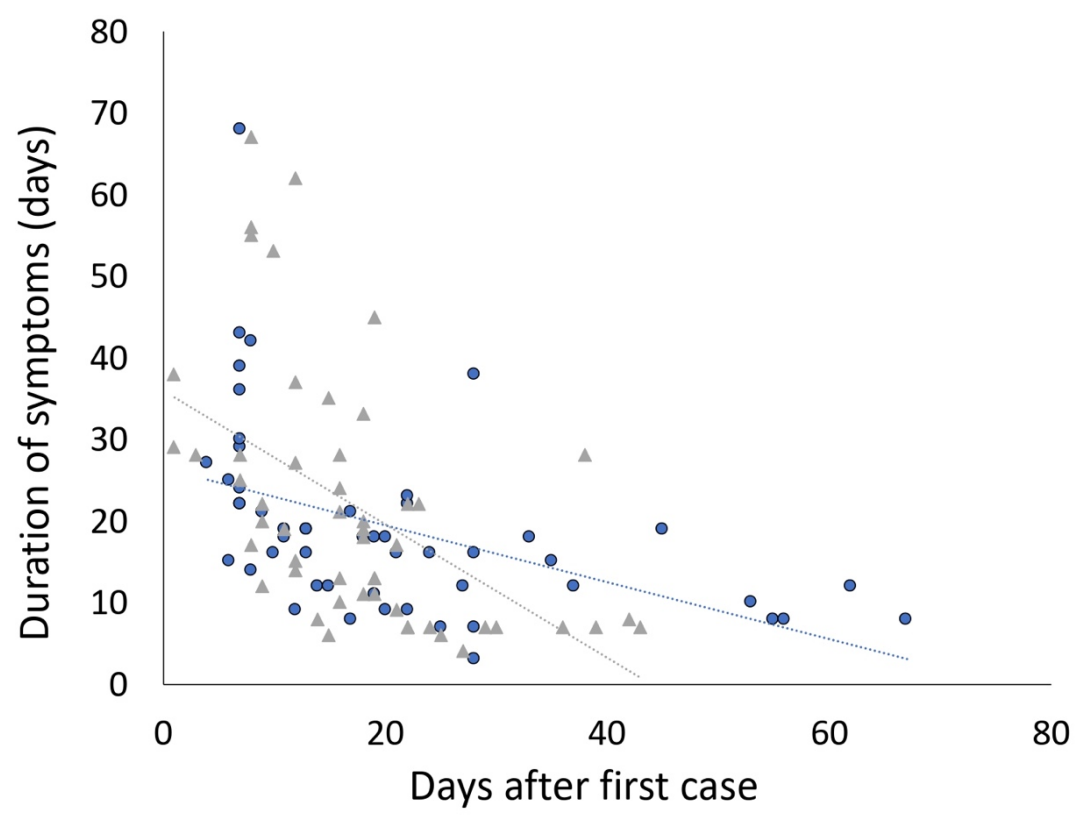

Figure 1. As time goes by, severity declines. Time (days after first case) correlates negatively with the duration of symptoms (in days) for patients younger than 60 years (circles) and older than 59 years (triangles) during the first COVID-19 outbreak in an isolated population.

Case fatality and thus possibly also disease severity of COVID-19 patients may be linked with length of follow up period (Rossi et al., 2020). However, in our small study population follow up is essentially continuous. Possible explanations for the observed decline in disease severity are improved anti-inflammatory treatments towards the end of the outbreak, changes in population susceptibility or pathogen virulence, less severe cases showing up later in time, or an effect of lockdown on viral burden or infective doses with subsequent effects of disease severity.

The COVID-19 pandemic presents numerous challenges to epidemiologists and health authorities. The ongoing de-escalation process in Spain will provide information on the most likely mechanisms explaining our observation. If the decline in severity is due to the lockdown, case severity and duration are expected to grow during de-escalation. In this case, only effective contact tracing followed by testing and case isolation should serve to control further outbreaks. However, if host- or pathogen-related factors are involved, this would represent good news since it might facilitate softer disease control interventions. Nevertheless, these observations will contribute to combating the current coronavirus pandemic, its aftermath and potential future clustered outbreaks or comparable healthcare emergencies.

Contributors 
FR and CG planned the study. Data were collected by FR and CG. Data analysis was led by LD and JdF. All authors interpreted the study findings, contributed to the manuscript, and approved the final version for publication.

Declaration of interests

We declare no competing interests.

Acknowledgments

This study had no specific funding. We would like to thank the local veterinarian Javier Camarena and the municipality of Horcajo de los Montes for information provided. We acknowledge UCLM support to Grupo SaBio.

Ethical statement

The authors confirm that the ethical policies of the journal, as noted on the journal's author guidelines page, have been adhered to. No ethical approval was required as this study was based on official records and anonymized. FR had full access to all the data in the study and all authors had final responsibility for the decision to submit for publication.

Data availability

The data that support the findings of this study are available from the corresponding author upon reasonable request.

\section{References}

Ding, X. et al. (2020). Chest CT findings of COVID-19 pneumonia by duration of symptoms. Eur. J. Radiol ., 127, 109009.

Huang, C. et al. (2020). Clinical features of patients infected with 2019 novel coronavirus in Wuhan, China. Lancet , 395 , 497- 506.

Rossi, P.G. et al. (2020). Case Fatality Rate in Patients With COVID-19 Infection and its relationship with length of follow up. J. Clin. Virol. , 128, 104415.

Shanks, D. et al. (2011). Determinants of mortality in naval units during the 1918-19 Influenza Pandemic. Lancet Infect. Dis. ,11, 793-9.

Wang, R. et al. (2020). Epidemiological and clinical features of 125 Hospitalized Patients with COVID-19 in Fuyang, Anhui, China. Int. J. Infect. Dis. , 95 , 421- 8.

Wu, J.T. et al. (2020a). Estimating clinical severity of COVID-19 from the transmission dynamics in Wuhan, China. Nat. Med .,26 , 506-10.

$\mathrm{Wu}$, Y. et al. (2020b). Effects of temperature and humidity on the daily new cases and new deaths of COVID-19 in 166 countries. Sci. Total Environ ., 729, 139051.

Zhang, G. et al. (2020). Clinical features and short-term outcomes of 221 patients with COVID-19 in Wuhan, China. J. Clin. Virol .,127, 104364. 